\title{
Impact of COVID-19 on Cardio-obstetric Risk Indicators: Future Directions
}

\author{
Prerna Goyal ${ }^{1}$ Shibba Takkar Chhabra² \\ ${ }^{1}$ Department of Medicine, RG Stone and Superspeciality Hospital, \\ Ludhiana, Punjab, India \\ 2Department of Cardiology, Dayanand Medical College and \\ Hospital, Hero DMC Heart Institute, Ludhiana, Punjab, India \\ Ind J Car Dis Wom:2020;5:209-215
}

\begin{abstract}
Address for correspondence Prerna Goyal, MD, Department of Medicine, RG Stone and Superspeciality Hospital, Ludhiana, Punjab, India (e-mail: drprerna156@gmail.com).
\end{abstract}

\begin{abstract}
Cardiovascular illness is one the foremost causes of death in females. The goal of preventive medicine is to identify and target high-risk females belonging to the younger age group in order to prevent future onset of cardiovascular pathologies. Certain obstetric complications like idiopathic preterm birth, preeclampsia (PE), gestational diabetes and abruptio placentae mark their presence in the checklist of risk indicators for cardiovascular diseases (CVD) among the female population. Previous severe acute respiratory syndrome-1 (SARS-1) infections recorded a significant impact on maternal and fetal outcomes. In this article, we aim to focus on the incidence of cardiovascular risk indicators with the incessant novel Coronavirus disease-19 (COVID-19) pandemic in pregnant women and to provide recommendations for venous thromboembolism (VTE) prophylaxis in infected females. As the disease is novel and gradually unfolding to clinicians globally, the data and the publications are also evolving. Studies on COVID-19 in pregnancy has shown an increase in the number of preterm births, and PE with some reports on vertical transmission, but no significant increase in miscarriage, still births and teratogenicity. Preterm births appear more likely to be iatrogenic rather than idiopathic, owing to severity of infection or maternal/fetal safety. Causal association of virus with PE needs further analysis by large population studies, as both have common overlapping clinical and laboratory parameters. Direct placental involvement by virus leading on to PE or infection itself simulating PE needs further explication. Assessment of placental function, histopathological examination, and measurement of antiangiogenic factors are some of the suggested measures to differentiate further. Peripartum cardiomyopathy in COVID-19 positive pregnant females has not yet been

Keywords

- cardiovascular

- preeclampsia

- pregnancy

- pre-term birth

- thromboembolism actively explored, particularly in cases of clinical deterioration. Practical utilization of handheld point-of-care echocardiography machines stand in need for diagnosing myocardial dysfunction in this pandemic. COVID-19 infection and pregnancy together may impart a greater thromboembolism risk due to their inherent hypercoagulable states. All pregnant females with COVID-19 infection are candidates for VTE prophylaxis, both antepartum and postpartum depending on the severity of illness.
\end{abstract}

\section{Review}

Pregnancy is a physiological state characterized by major hemodynamic changes throughout pregnancy till labor and postpartum period. Clinical spectrum of cardiovascular diseases (CVD) in pregnancy can vary from worsening of

known cardiac illness or unveiling of subclinical problem to new onset complications. Also, pregnancy offers a window of opportunity to cardiovascular clinicians to screen for certain adverse obstetric outcomes that have potential to predict future risk of CVD. Hypertensive disorders of pregnancy (HDP), gestational diabetes mellitus, idiopathic preterm 
birth, and intrauterine growth retardation are some of the predesignated risk indicators for future onset of CVD., ${ }^{1,2}$

As the novel Coronavirus disease-19 (COVID-19) outbreak unfolds, cardiac involvement has become an area of interest for clinicians all over the world. There is emerging data on impact of severe acute respiratory syndrome coronavirus-2 (SARS-CoV-2) on various maternal obstetric complications and neonatal outcomes in pregnant women. This element of COVID-19 in pregnancy is of major concern, especially when previous studies confirm a high incidence of adverse obstetric outcomes with SARS-1,3,4 This article aims to discuss in detail the various cardiovascular risk indicators in pregnancy with COVID-19 infection, so as to help primary care providers for optimal obstetrics care and provide directions for future surveillance of CVD. It also focuses on guidelines for the management of venous thromboembolism (VTE) in pregnancy.

\section{Premature Birth (PTB)}

PTB and very preterm births are defined as delivery at $<37$ weeks and $<32$ weeks of gestation, respectively. Idiopathic PTB increases the future risk of CVD by $40 \%$ and very preterm delivery almost doubles the cardiovascular risk. ${ }^{5}$

Any infective pathology or inflammatory process can increase the risk of PTB. A PTB may be spontaneous/idiopathic when it happens itself or iatrogenic when delivery is induced early for maternal and neonatal safety. Previous SARS infections have been associated with higher PTB. Reports of COVID-19 in pregnancy also found increased number of preterm deliveries in various studies. ${ }^{6,7}$ But whether this reported high incidence in COVID-19 is primarily due to spontaneous onset of labor or secondary to elective induction of delivery due to severity of illness/maternal respiratory distress needs to be explored. Much of clinical data in COVID-19 suggest increase in number of PTB mainly due to maternal and fetal compromise. One UK-based cohort study suggested that $80 \%$ had iatrogenic PTBs; $48 \%$ attributed to maternal, $14 \%$ to fetal, and $18 \%$ to miscellaneous obstetric indications. ${ }^{8}$ Similar data has been published by a US cohort study that reported $88 \%$ with critical infection had iatrogenic PTB. ${ }^{9}$

Reported effects of COVID-19 on other obstetric outcomes do not show significant rise in miscarriage rate, stillbirths, teratogenicity, and neonatal morbidity despite few reports of vertical transmission. Thus, for an infection, which seems to have a similar clinical course as in normal pregnant population in all aspects, it should not be expected to behave differently for occurrence of idiopathic PTB. The potential explanations for insignificant effects on maternal obstetric outcomes could be improved hygiene, confinement to homes, lesser societal interactions, better air quality, and changing habits adopted during lockdown. In short, it is too early to believe the causal association of COVID-19 with idiopathic PTB. So, whether COVID-19 infected women with history of PTB should be put on the surveillance list of future assessment of CVD is still unclear.

\section{Hypertensive Disorders of Pregnancy}

Hypertension in pregnancy is one of the most commonly encountered cardiovascular complications. It comprises chronic hypertension, gestational hypertension, preeclampsia (PE) with or without preexisting hypertension. Definition of hypertension during pregnancy includes systolic blood pressure (BP) of $140 \mathrm{~mm} \mathrm{Hg}$ or higher, diastolic BP of $90 \mathrm{~mm}$ $\mathrm{Hg}$ or higher, or both. PE is a multiorgan disease process characterized by newly discovered hypertension or aggravation of previous hypertension with newly developed proteinuria or aggravation of previous proteinuria with or without end organ manifestations. HELLP syndrome (hemolysis, elevated liver enzymes, and low platelets) is a more severe form of PE, complicating approximately $20 \%$ of severe PE cases.

\section{COVID-19 and PE Risk Association}

The main pathophysiology in PE originates around antiangiogenic mediators and placental oxidative stress, leading to endothelial damage, while SARS-CoV-2 primarily affects renin-angiotensin system, leading to vasoconstriction. SARS-CoV-2 infection in pregnant women has been reported to be associated with increased risk of PE in a few case series and small cohort studies. ${ }^{10}$

In fact, many of the symptomatology and laboratory parameters for COVID-19 can simulate the findings observed in severe PE. Simultaneous presence of hypertension, proteinuria, coagulopathy, or liver injury in both may pose diagnostic challenges..$^{11}$ Therefore, there might be high chances of misdiagnosing or overdiagnosing PE in many such patients. One such interesting observation has been made by Mendoza and colleagues who reported PE-like syndrome in six out of eight pregnant patients with severe SARS-CoV-2 pneumonia. ${ }^{12}$ However, only one out of six had evidence of placental dysfunction on Doppler examination and biochemical analysis. One more study has reported histopathological changes in placenta from COVID-19 patients postdelivery. ${ }^{13}$ Features of impaired placental oxygenation and poor maternal vascular perfusion were found in all 16 placentas studied, but fortunately all had normal uncomplicated deliveries, and only one had clinical evidence of antenatal high BP. These observations provide insight into the presence of subclinical placental changes compatible with HDPs in COVID-19 patients.

All these findings stand in need for future research among a larger population group. Also, whether reported high incidence of PE in severe COVID-19 infections is a true reflection of placental dysfunction due to virus itself or it is just a part of the disease spectrum needs to be answered with more robust data.

\section{Differentiation between Severe COVID-19 Infection and Preeclampsia (PE)}

Present history of fever in COVID-19 infection, or previous history of hypertension/predominant finding of high $\mathrm{BP}$ in PE, may help in differentiation to some extent on 
clinical grounds. Doppler assessment (uterine artery pulsatility index) and estimation of angiogenic factors (soluble fms-like tyrosine kinase-1/placental growth factor, lactate dehydrogenase) are few of the diagnostic measures to distinguish severe COVID-19 infection from true PE. ${ }^{12}$

\section{Antenatal Home BP Monitoring-Are They Reliable?}

One of the best indicators of optimal obstetric care is precise measurement of BP. With COVID-19 upsurge, all pregnant women should be advised to minimize their clinic visits and encouraged to home record their BP on a daily basis at the same time of the day with the help of validated home devices. US Food and Drug Administration has recently given acceptance to the home application of noninvasive maternal monitoring devices for conditions unrelated to COVID-19. ${ }^{14}$ Written instructions on the important signs and symptoms of disease progression along with emergency contact information can be provided to all pregnant women.

\section{Aspirin as PE Prophylaxis in COVID-19 Pregnant Women}

ACOG and Society of Maternal and Fetal Medicine (SMFM) recommend low-dose aspirin ( $81 \mathrm{mg} /$ day) as a prophylactic measure for PE to all high-risk pregnant women between 12 to 28 weeks of gestation till delivery. ${ }^{15}$ All initial trials of nonsteroidal anti-inflammatory drugs (NSAIDs) in COVID-19 came out with some negative results. Recommendation for aspirin prophylaxis in high-risk pregnant women with suspected or confirmed COVID-19 is to individualize the risk-benefit ratio, depending on the gestational age and severity of illness. For example, it is doubtful to have any benefits of aspirin in very severe infections or near term. ${ }^{16}$

\section{Magnesium Sulfate (MgSO4) for Severe PE in COVID-19 Pregnant Women}

MgSO4 is routinely recommended for fetal neuroprotection and seizure prophylaxis in PE management protocol. SMFM and Society for Obstetric Anesthesia and Perinatology suggests that beneficial effects of $\mathrm{MgSO} 4$ should be weighed against its potential risks of maternal respiratory depression and renal injury. ${ }^{17}$

A few recommendations by SMFM consider withholding or dose adjusting $\mathrm{MgSO} 4$ among the following patients:

- Presence of severe respiratory distress, need for ventilator support, and/or ongoing benzodiazepine therapy.

- Acute kidney injury.

- Anticipated low risk of preterm birth. ${ }^{18}$

\section{Timing of Delivery}

Current guidelines from ACOG and SMFM for medical management of severe PE advocate delivery anytime after 34 weeks of gestation. With the outbreak of COVID-19, an early delivery by 30 to 32 weeks in patients with severe PE can be planned, keeping in mind that PE-like clinical picture can also be produced due to COVID-19 itself. Thus, an early delivery should not be contemplated in haste, as there are chances of clinical improvement with the resolution of severe pneumonia.

\section{Does Past History of PE Increase the Present Risk of Getting COVID-19?}

Contrary to the previous reports with H1N1 infection, none of the available data support higher risk of getting COVID-19 in such patients. Women who are currently normotensive or having well-controlled BP with drugs are not predisposed to SARS-CoV-2 infection. Uncontrolled persistent high BP in postpartum period may predispose to higher risk of getting infection.

\section{Peripartum Cardiomyopathy in Pregnancy with COVID-19}

Acute cardiac insult secondary to nonischemic myocardial injury due to cytokine storm is the most common documented cardiac presentation in COVID-19. ${ }^{19,20}$ An increased incidence of stress/Takotsubo cardiomyopathy (TCM) had been found in a cohort study of 1914 patients who presented with acute coronary syndrome during the COVID-19 pandemic as compared with the prepandemic period. ${ }^{21}$ New onset cardiomyopathy has also been demonstrated in 33\% of nonpregnant COVID-19 patients who required intensive care. ${ }^{22}$ Viral genome has also been demonstrated in myocardial tissue of such patients in one study. ${ }^{23}$

But effects of SARS-CoV-2 on myocardial function or incidence of peripartum cardiomyopathy (PPCM) in pregnant females remain largely unknown to date. PPCM is a type of idiopathic dilated cardiomyopathy with onset near term till 5 months postdelivery. There is currently no documented cohort or population-based studies on incidence of PPCM with SARS-CoV-2 in pregnant women. Few of the data available so far is only in the form of case studies and case series.

There is one paper, which described two cases of cardiomyopathy in SARS-CoV-2 positive pregnant women. ${ }^{24}$ The diagnosis was made after getting echocardiogram of pregnant mothers who had rapid progressive down course in the setting of elevated troponin-I. This report also suggested the role of performing echocardiography in particular subsets of COVID-19 positive pregnant patients with severe pneumonia and progressive respiratory distress requiring high-oxygen support to diagnose underlying myocardial dysfunction. European society of cardiology advisory on management of CVD in COVID-19 pandemic restricts the routine usage of transthoracic echocardiography to minimize the viral spread. ${ }^{25}$

The major reasons for under-reporting of PPCM in COVID-19 infected patients could be ascribed to lack of resources, unavailability of echocardiograms, and nonperformance of cardiac markers, owing to poor suspicion of myocardial dysfunction in the setting of severe COVID-19 pneumonias. For echocardiography in emergency need, use of small handheld point-of-care devices instead of traditional large machines, which are easy to decontaminate, should be stressed upon during the COVID-19 pandemic. In short, more data is needed 
to determine the true incidence of cardiomyopathy in pregnancy and postpartum period secondary to COVID-19.

\section{Venous Thromboembolism (VTE) in COVID-19 Pregnancy}

As SARS-CoV-2 has been designated as hypercoagulable state, various international bodies recommend routine pharmacologic VTE prophylaxis in all hospitalized patients with COVID-19 unless contraindicated. Pregnancy together with COVID-19 infection may pose a distinctive increased thrombotic risk due to dual physiological and virus-related hypercoagulability. ${ }^{26}$

\section{Recommendations for VTE Prophylaxis in COVID-19 Positive Pregnant Women}

The various statements issued are as follows:

1) American College of Obstetricians and Gynecologists (ACOG) advises antenatal thromboprophylaxis in all such patients, particularly in severe/critical disease. ${ }^{27}$

2) Royal College of Obstetricians and Gynecologists (RCOG) recommends that pregnant women with mild infection and on home isolation should undergo clinical VTE risk assessment via telehealth or office visit, and thromboprophylaxis should be considered on basis of their risk score. ${ }^{28}$ Multiple risk assessment models are available to help estimate the risk of VTE in pregnant population. One such freely available software tool based on international guidelines is AnticoagObs (http://www.anticoagulationinpregnancy.com/).29,30 Homeconfined patients can be taught technique of self-injection by telehealth or a video link and prescription can be sent by post.

3) Queensland Health strongly recommends antenatal VTE prophylaxis in all pregnant women with suspected/confirmed COVID-19 infection, even in the absence of other risk factors. ${ }^{31}$
Based on all available resources, clinical practice guidelines on VTE prophylaxis can be as summarized as below ( - Table 1).

- Apart from these, all pregnant women who are already on anticoagulation because of some other reason should continue taking it

- Also, all obstetric patients with COVID-19 who got admitted to the hospital for management of any of their obstetric or medical disorder should be given VTE prophylaxis unless birth is expected within 12 hours.

In all these recommendations, it should be remembered that third trimester and puerperium are designated highrisk periods for VTE and infection in these periods should be strongly considered for prophylaxis.

\section{Venous Thromboembolism in Postpartum Period}

RCOG and Queensland Health Clinical Guidance consider universal postpartum VTE prophylaxis for all suspected/ confirmed COVID-19 patients ${ }^{28,31}$. RCOG also recommends prophylactic low-molecular weight heparin (LMWH) for $\geq 10$ days upon discharge regardless of mode of birth to be extended till 6 weeks in high-risk women ${ }^{29}$. For COVID-19 positive pregnant patients who did not receive antepartum pharmacologic prophylaxis, postpartum prophylaxis is not required in mild or asymptomatic infections with uncomplicated deliveries.

Clinical practice guidance for VTE prophylaxis in postpartum period has been summarized in - Table 1 .

\section{Choice of Anticoagulation}

Anticoagulation in pregnant women needs a careful approach. The Seventh American College of Chest Physicians Consensus Conference on Antithrombotic Therapy recommends three types of anticoagulation therapy during pregnancy. ${ }^{32}$ In pregnant women for VTE prophylaxis, heparin is considered

Table 1 Proposed guidelines for prophylaxis of VTE in pregnant women with COVID-19 infection

\begin{tabular}{|l|l|l|l|}
\hline \multicolumn{3}{|c|}{ Asymptomatic SARS-CoV-2 infection } \\
\hline \multicolumn{3}{|c|}{ Assess for VTE risk factors, advice home isolation, provide instructions for home monitoring } \\
\hline \multicolumn{3}{|c|}{ Symptomatic SARS-CoV-2 infection } \\
\hline $\begin{array}{l}\text { Suspect (symptoms con- } \\
\text { sistent with COVID-19) }\end{array}$ & $\begin{array}{l}\text { Wait for SARS-CoV-2 report, reassess VTE risk factors, } \\
\text { monitor at home every 48-72 hours, instruct to report } \\
\text { if any symptoms appear }\end{array}$ & $\begin{array}{l}\text { Same as management of SARS-CoV-2 positive } \\
\text { patients }\end{array}$ \\
\hline Antenatal & $\begin{array}{l}\text { Prophylactic } \\
\text { LMWH } \times \\
2 \text { weeks may be } \\
\text { considered after } \\
\text { risk assessment }\end{array}$ & $\begin{array}{l}\text { Prophylactic LMWH x 2 weeks and } \\
\text { up to 7 days post discharge }\end{array}$ & $\begin{array}{l}\text { Prophylactic LMWH at admission and 1-month } \\
\text { postdischarge, if started in third trimester extend } \\
\text { till delivery plus 6 weeks postpartum }\end{array}$ \\
\hline $\begin{array}{l}\text { Prophylactic } \\
\text { LMWH } \times \\
2 \text { weeks in low } \\
\text { risk and 6 weeks } \\
\text { in high risk } \\
\text { group }\end{array}$ & $\begin{array}{l}\text { Prophylactic LMWH } \times 2 \text { weeks and } \\
\text { up to 7 days post discharge, extend } \\
\text { till 6 weeks in high-risk group }\end{array}$ & Prophylactic LMWH for at least 6 weeks \\
\hline
\end{tabular}

Abbreviations: LMWH, low-molecular weight heparin; VTE, venous thromboembolism. 
to be the anticoagulant of choice, owing to its fetal safety. Moreover, heparin has additional anti-inflammatory properties that may help in improved clinical outcomes.

- LMWH: It produces more predictable anticoagulation. Chances of thrombocytopenia and maternal osteoporosis are less. Preferred if delivery is not expected within 24 hours and in postpartum period.

\section{- Unfractionated heparin (UFH):}

1. Preferred where faster reversal of anticoagulation is required, for example, if delivery or any invasive procedure is expected within 12 to 24 hours.

2. Preferred for patients on renal replacement therapy or creatinine clearance $(\mathrm{CrCl})<15 \mathrm{~mL} / \mathrm{min}$.

UFH needs dose monitoring, according to activated partial thromboplastin time (aPTT) and/or anti-Xa levels. Anti-Xa levels are indicated in patients with suspected heparin resistance or with baseline prolonged aPTT. Adverse effects reported are maternal osteoporosis, hemorrhage, thrombocytopenia, or thrombosis. In fact, heparin-induced thrombocytopenia (HIT) may confound the laboratory findings of COVID-19. The "4Ts" scoring system or presence of HIT antibodies may guide in differential diagnosis. ${ }^{33}$

\section{Indications for Full-dose Anticoagulation in COVID-19 Positive Pregnant Patients}

Direct clinical data comparing different intensity of anticoagulation (prophylactic, intermediate, or therapeutic) in such patients is lacking. Some experts advocate measuring levels of D-dimer and/or C-reactive protein to guide dose requirements. However, in pregnancy, D-dimer levels are not considered a good guide for dose titration, as there is physiological rise in D-dimer in pregnancy. Any theoretical benefits of full-dose anticoagulation in COVID-19 positive pregnant patients should be weighed against potential risk profile associated with high doses. As these patients are at relatively high risk of preterm births, therapeutic anticoagulation may pose an increased risk for postpartum hemorrhage. If need be, UFH should be the drug of choice for therapeutic anticoagulation. A few of the indications are as follows:

- Direct definite evidence of VTE on standard imaging.

- Any indirect evidence of VTE-demonstration of deep vein thrombosis on compression ultrasonography of lower limbs or presence of clot in main pulmonary artery on transthoracic echocardiography.

- Sudden unexplained respiratory distress, which is not attributable to cardiac cause/fluid overload/acute respiratory distress syndrome (ARDS), especially in the setting of raised fibrinogen and/or D-dimer levels.

- Recurrent clotting of intravascular access devices despite prophylactic anticoagulation.

- Critically ill patients. ${ }^{18}$

Dosage schedule of LMWH and UFH in pregnancy, according to the desired intensity/level of anticoagulation, is shown in - Table 2. UFH and LMWH need modification in cases with renal impairment as per standard guidelines. ${ }^{34}$

To conclude, although few of the obstetric risk indicators like PTB and PE have shown an increased incidence in pregnant females with COVID-19 infection, but further large studies are required to ascertain the cause-effect relationship. Cardiomyopathy in pregnant women with COVID-19 is most probably under reported due to echocardiography constraints and remote healthcare provision in the COVID era. Currently, it is too early to foresee the slope of trajectory of cardiovascular risk indicators in pregnancy with COVID-19 infection, and whether these patients should be actively targeted for preventive medicine needs to be explored further.

Table 2 Heparin dosage schedule during pregnancy

\begin{tabular}{|l|l|l|l|l|}
\hline Heparin & & Prophylactic & Intermediate & Therapeutic \\
\hline LMWH & Enoxaparin & $40 \mathrm{mg}$ SC once daily & $\begin{array}{l}40 \mathrm{mg} \text { SC once daily, increase as pregnancy } \\
\text { progress to } 1 \mathrm{mg} / \mathrm{kg} \text { once daily }\end{array}$ & 1 mg/kg SC every 12 hourly \\
\cline { 2 - 5 } & Dalteparin & $\begin{array}{l}5000 \text { units SC once } \\
\text { daily }\end{array}$ & $\begin{array}{l}5000 \text { units SC once daily, increase as pregnancy } \\
\text { progress to } 100 \text { units/kg once daily }\end{array}$ & $\begin{array}{l}100 \text { units/kg SC every } 12 \\
\text { hourly }\end{array}$ \\
\hline UFH & $\begin{array}{l}5000 \text { units SC every } \\
12 \text { hourly }\end{array}$ & $\begin{array}{l}\text { First trimester-5000-7500 units SC } 12 \text { hourly } \\
\text { Second trimester-7500-10,000 units SC } 12 \text { hourly } \\
\text { Third trimester-10,000 units SC 12 hourly }\end{array}$ & $\begin{array}{l}\text { Continuous IV infusion or SC } \\
\text { every } 12 \text { hourly, titrate to keep } \\
\text { aPTT in therapeutic range }\end{array}$ \\
\hline
\end{tabular}

Abbreviations: aPTT, activated partial thromboplastin time; LMWH, low-molecular weight heparin; UFH, unfractionated heparin. 


\section{Conflicts of Interest}

None declared.

\section{References}

1 Ray JG. Metabolic syndrome and higher risk of maternal placental syndromes and cardiovascular disease. Drug Dev Res 2006;67:607-611

2 Retnakaran R, Qi Y, Connelly PW, Sermer M, Hanley AJ, Zinman B. The graded relationship between glucose tolerance status in pregnancy and postpartum levels of low-density-lipoprotein cholesterol and apolipoprotein B in young women: implications for future cardiovascular risk. J Clin Endocrinol Metab 2010;95(9):4345-4353

3 Wong SF, Chow KM, Leung TN, et al. Pregnancy and perinatal outcomes of women with severe acute respiratory syndrome. Am J Obstet Gynecol 2004;191(1):292-297

4 Lam CM, Wong SF, Leung TN, et al. A case-controlled study comparing clinical course and outcomes of pregnant and non-pregnant women with severe acute respiratory syndrome. BJOG 2004;111(8):771-774

5 Tanz LJ, Stuart JJ, Williams PL, et al. Preterm Delivery and Maternal Cardiovascular Disease in Young and Middle-Aged Adult Women. Circulation 2017;135(6):578-589

6 Dashraath P, Wong JL, Lim MXK, et al. Coronavirus disease 2019 (COVID-19) pandemic and pregnancy. Am J Obstet Gynecol 2020;222(6):521-531

7 Panahi L, Amiri M, Pouy S. Risks of Novel Coronavirus Disease (COVID-19) in Pregnancy; a Narrative Review. Arch Acad Emerg Med 2020;8(1):e34

8 Knight M, Bunch K, Vousden N, et al; UK Obstetric Surveillance System SARS-CoV-2 Infection in Pregnancy Collaborative Group. Characteristics and outcomes of pregnant women admitted to hospital with confirmed SARS-CoV-2 infection in UK: national population based cohort study. BMJ 2020;369: $\mathrm{m} 2107$

9 Pierce-Williams RAM, Burd J, Felder L, et al Clinical course of severe and critical coronavirus disease 2019 in hospitalized pregnancies: a United States cohort study. Am J Obstet Gynecol MFM2020 (e-pub ahead of print). doi: https://doi. org/10.1016/j.ajogmf.2020.100134

10 COVID-19 infection was associated with higher rate (and pooled proportions) of preterm birth, preclampsia, cesarean, and perinatal death. Am J ObstetGynecol MFM 2020;2(2):100107

11 American College of Obstetricians and GynecologistsTask Force on Hypertension in Pregnancy. Hypertension in pregnancy. Report of the American College of Obstetricians and Gynecologists' Task Force on Hypertension in Pregnancy. Obstet Gynecol 2013;122(5):1122-1131

12 Mendoza M, Garcia, -, Ruiz I, Maiz N, et al. Pre-eclampsia-like syndrome induced by severe COVID-19: a prospective observational study. BJOG 2020;(e-pub ahead of print). doi: https:// doi.org/10.1111/1471-0528.16339

13 Shanes ED, Mithal LB, Otero S, Azad HA, Miller ES, Goldstein JA. Placental Pathology in COVID-19. Am J Clin Pathol 2020;154(1):23-32

14 Enforcement Policy for Non-Invasive Fetal and Maternal Monitoring Devices Used to Support Patient Monitoring During the Coronavirus Disease. 2019 (COVID-19) Public Health Emergency. Guidance for Industry and Food and Drug Administration Staff. Available at: https://www.fda. gov/regulatory-information/search-fda-guidance-documents/enforcement-policy-non-invasive-fetal-andmaternal-monitoring-devices-used-support-patient. Accessed April 24, 2020
15 American College of Obstetricians and Gynecologists (ACOG) Committee Opinion No. 743. Low-dose aspirin use during pregnancy. Obstet Gynecol 2018;132:e44-e52

16 European Medicines Agency. EMA gives advice on the use of non-steroidal anti-inflammatories for COVID-19. Available at: https://www.ema.europa.eu/en/news/ema-gives-adviceuse-non-steroidal-anti-inflammatories-covid-19. Accessed March 19, 2020.

17 Miller E, Leffert L, Landau R. Society for Maternal-Fetal Medicine and Society for Obstetric and Anesthesia and Perinatology Labor and Delivery COVID-19 Considerations. Available at: https://s3.amazonaws.com/cdn.smfm.org/media/2402/ SMFM-SOAP_COVID_LD_Considerations_-_revision_616-20_PDF.pdf. Accessed June 25, 2020

18 Halscott T, Vaught J. Society for Maternal-Fetal Medicine Management Considerations for Pregnant Patients With COVID-19. Available at: https://s3.amazonaws.com/cdn.smfm.org/media/2336/SMFM_COVID_ Management_of_COVID_pos_preg_patients_4-30-20_final. pdf. Accessed June 25, 2020.

19 Bansal M. Cardiovascular disease and COVID-19. Diabetes Metab Syndr 2020;14(3):247-250

20 Guan WJ, Ni ZY, Hu Y, et al; China Medical Treatment Expert Group for Covid-19. Clinical characteristics of coronavirus disease 2019 in China. N Engl J Med 2020;382(18):1708-1720

21 Jabri A, Kalra A, Kumar A, et al. Incidence of stress cardiomyopathy during the Coronavirus disease 2019 pandemic. JAMA Netw Open 2020;3(7):e2014780

22 Guo T, Fan Y, Chen M, et al. Cardiovascular implications of fatal outcomes of patients with coronavirus disease 2019 (COVID-19) JAMA Cardiol 2020;5(7):1-8

23 Kühl U, Pauschinger M, Noutsias M, et al. High prevalence of viral genomes and multiple viral infections in the myocardium of adults with "idiopathic" left ventricular dysfunction. Circulation 2005;111(7):887-893

24 Juusela A, Nazir M, Gimovsky M. Two cases of coronavirus 2019-related cardiomyopathy in pregnancy. Am J Obstet Gynecol MFM 2020;2(2):100113

25 The European Society for Cardiology. ESC Guidance for the Diagnosis and Management of CV Disease during the COVID-19 Pandemic. Available at: https://www.escardio.org/Education/ COVID-19-and-Cardiology/ESC-COVID-19-Guidance. Accessed June 10, 2020

26 Thrombosis UK. Practical guidance for the prevention of thrombosis and management of coagulopathy and disseminated intravascular coagulation of patients infected with COVID-19. Available at: https://thrombosisuk. org/covid-19-thrombosis. php. Accessed April 07, 2020.

27 American College of Obstetricians and Gynecologists. COVID-19 FAQs for Obstetricians-Gynecologists, Obstetrics. Available at: https://www.acog.org/clinical-information/physician-faqs/covid-19-faqs-for-ob-gyns-obstetrics. Accessed June 25, 2020

28 Royal College of Obstetricians and Gynaecologists (RCOG). Coronavirus (COVID-19) Infection in Pregnancy. Available at: https://www.rcog.org.uk/coronavirus-pregnancy. Accessed June 25, 2020

29 Royal College of Obstetricians and Gynaecologists. Reducing the Risk of Venous Thromboembolism during Pregnancy and the Puerperium. (Green-top Guideline No. 37a). Available at: https://www.rcog.org.uk/globalassets/documents/guidelines/ gtg-37a.pdf. Accessed June 10, 2015

30 Bates SM, Greer IA, Middeldorp S, Veenstra DL, Prabulos AM, Vandvik PO. VTE, thrombophilia, antithrombotic therapy, and pregnancy. Antithrombotic Therapy and Prevention of Thrombosis, 9th ed: American College of Chest Physicians Evidence-Based Clinical Practice Guidelines. Chest 2012;141(2) (Suppl):e691S-e736S 
31 Queensland Health Clinical Guidelines. Maternity Care for Mothers and Babies during the COVID-19 Pandemic. Available at: https://www.health.qld.gov.au/_data/assets/ pdf_file/0033/947148/g-covid-19.pdf. Accessed July 17, 2020

32 Bates SM, Greer IA, Hirsh J, Ginsberg JS. Use of antithrombotic agents during pregnancy: the Seventh ACCP Conference on Antithrombotic and Thrombolytic Therapy. Chest 2004;126(3, Suppl):627S-644S

33 Cuker A, Gimotty PA, Crowther MA, Warkentin TE. Predictive value of the 4Ts scoring system for heparin-induced thrombocytopenia: a systematic review and meta-analysis. Blood 2012;120(20):4160-4167

34 Hughes S, Szeki I, Nash MJ, Thachil J. Anticoagulation in chronic kidney disease patients-the practical aspects. Clin Kidney J 2014;7(5):442-449 